\title{
RELAÇÕES ENTRE COMPOSIÇÃO E MICROESTRUTURA E SUSCETIBILIDADE À DEGRADAÇÃO MECÂNICA DE PELOTAS QUEIMADAS DE MINÉRIO DE FERRO PARA REDUÇÃO DIRETA*
}

\author{
Pedro Porto Silva Cavalcanti ${ }^{1}$ \\ Túlio Moreira Campos ${ }^{2}$ \\ Maciel Bianchi Rodrigues ${ }^{3}$ \\ Ana Maria Guilherme Bailon 4 \\ Luís Marcelo Marques Tavares ${ }^{5}$
}

\section{Resumo}

A suscetibilidade à degradação mecânica de pelotas de minério de ferro é diretamente influenciada tanto pela composição quanto pela sua estrutura. O trabalho analisou criticamente essas relações para pelotas queimadas para a redução direta, por meio de análises detalhadas com quatro tipos distintos de pelotas, que incluíram análises químicas e mineralógicas, medidas de porosidade e distribuição de tamanhos de poros por tomografias de raios $\mathrm{X}$, distribuição de poros por região da pelota por meio de microscopia, da dureza, bem como de resistência à compressão, abrasão, bem como um novo método de avaliação da resistência à fragmentação por abrasão. O trabalho mostra que há, de fato, forte correlação entre a suscetibilidade à degradação e várias dessas características analisadas. Forte correlação foi observada entre a porosidade e o tamanho máximo de poros. Boa correspondência qualitativa foi observada entre o acabamento superficial e a resistência à compressão. Resultados de um novo teste proposto de suscetibilidade à fragmentação superficial sugerem que a porosidade total e a mineralogia exercem pouca influência nesse comportamento, enquanto as características superficiais, como o acabamento e a microdureza exercem uma influência direta nesse mecanismo.

Palavras-chave: Mineralogia; Porosidade; Degradação; Pelotas

\section{RELATIONSHIPS BETWEEN COMPOSITION AND MICROSTRUCTURE AND SUSCEPTIBILITY TO MECHANICAL DEGRADATION OF BURNED DIRECT REDUCTION IRON ORE PELLETS}

\section{Abstract}

Susceptibility to mechanical degradation of iron ore pellets is directly influenced by the composition and the structure. This job critically examined these relationships to four different types of burned pellets, for direct reduction, through detailed analysis, which included chemical and mineralogical analysis, porosity measurements, pore size distribution by X-ray tomography, pellet porosity area distribution by microscopy, hardness, compression resistance, abrasion, as well a new methodology for evaluating abrasion resistance fragmentation. This work shows that there is, indeed, a strong correlation between susceptibility to mechanical degradation and several of these characteristics analyzed. A strong correlation was observed between the porosity and the maximum pore size. Good qualitative correlation was observed between the surface characteristics and the compression resistance. Results of a new proposed test, from susceptibility to surface fragmentation, suggest that the total porosity and the mineralogy have little influence in this behavior, as the surface characteristics such as finish and hardness exert a direct influence on this mechanism. Keywords: Mineralogy; Porosity; Degradation; Pellets.

1 Engenheiro Metalurgista, M.Sc., Aluno de Doutorado, Laboratório de Tecnologia Mineral, COPPE/UFRJ, Rio de Janeiro, RJ, Brasil.

2 Graduando em Engenharia Metalúrgica, Aluno de Iniciação Científica, Laboratório de Tecnologia Mineral, COPPE/UFRJ, Rio de Janeiro, RJ, Brasil.

3 Engenheiro Metalurgista, Analista de Marketing. Samarco Mineração S.A., Anchieta, ES, Brasil, Aluno de Mestrado, Laboratório de Tecnologia Mineral, COPPE/UFRJ.

4 Técnico em Metalurgia, Analista de Marketing.. Samarco Mineração S.A., Anchieta, ES, Brasil.

5 Eng. de Minas, Ph.D., Professor Titular, Laboratório de Tecnologia Mineral, COPPE/UFRJ, RJ, Brasil. 


\section{INTRODUÇÃO}

A degradação de pelotas queimadas de minério de ferro ocorre, principalmente, durante as etapas de manuseio e transporte, que geram quantidades apreciáveis de material fino (partículas de tamanhos menores que, aproximadamente, 6 milímetros), prejudicial aos processos de redução dos fornos siderúrgicos. Esses finos são removidos da alimentação dos fornos, por peneiramento, e vendidos a custos marginais pelas siderúrgicas que não dispõem de unidades de sinterização, traduzindo-se em prejuízos. Dessa forma, as pelotizadoras realizam um controle rigoroso do tamanho e qualidade das pelotas durante o processo de produção. A qualidade é medida de acordo com as propriedades das pelotas, sendo a resistência mecânica uma das mais importantes.

A integridade física das pelotas, necessária para resistir aos esforços mecânicos que ocorrem durante o manuseio e transporte está relacionada a fatores de natureza química e físico-química, pois as pelotas de minério de ferro são produzidas a partir da adição de alguns insumos ao concentrado de minério de ferro propriamente dito. $A$ composição química da maioria das pelotas queimadas, produzidas no Brasil, é de cerca de $95 \%$ de minerais de ferro $\left(\mathrm{Fe}_{2} \mathrm{O}_{3}\right.$ e $\left.\mathrm{Fe}_{3} \mathrm{O}_{4}\right)$ e $5 \%$ de outros compostos que podem ser resumidos em $\mathrm{SiO}_{2}, \mathrm{Al}_{2} \mathrm{O}_{3}, \mathrm{CaO}$ e $\mathrm{MgO}$. Com essa composição química fortemente controlada, sua resistência mecânica está associada, em grande parte, à sua estrutura interna, que após a etapa de queima é constituída de três porções distintas: grãos de minério sinterizados, fases escorificadas e poros [1]. Sendo assim, a análise estrutural (análises mineralógica e de porosidade) de pelotas, após sua queima, oferece indícios sobre a sua resistência mecânica potencial. Isso se deve ao fato que a resistência à compressão das pelotas é função direta da coesão da massa global das pelotas, dada pela eficiência da sinterização e do crescimento dos grãos de hematita, pela quantidade e composição das fases escorificadas presentes entre os grãos e no interior de alguns poros, pela quantidade e tamanho dos poros e da proporção de cavidades não preenchidas [1].

O crescimento dos grãos de hematita é resultado de uma boa etapa de queima, já que sua presença nas pelotas queimadas se deve à hematita primária ou à hematita secundária. A hematita primária é proveniente do próprio minério, enquanto a hematita secundária é formada a partir da oxidação da magnetita, seja a magnetita também proveniente do minério ou aquela formada pela redução da hematita, sendo posteriormente reoxidada em hematita [2]. Inclusive, caso a etapa de queima não tenha sido realizada de maneira eficiente, podem ainda ser encontrados elevados teores de magnetita residual na pelota, que pode ter um efeito deletério na resistência física da pelota [2]. A magnetita residual pode ser originária de queima a baixas temperaturas ou então pelo uso de temperaturas excessivamente altas, gerando queima excessiva e redução da hematita em magnetita.

A porosidade é outra característica importante de pelotas queimadas. Ela pode ser afetada pela mineralogia do pellet feed, pela dosagem de carvão e calcário na pelota verde e pela composição química final das pelotas. A composição química pode afetar a formação da escória, já que uma quantidade maior de fases escorificadas pode bloquear alguns poros, limitando o contato gás redutor com o sólido, diminuindo a redutibilidade das pelotas [1]. A presença da fase líquida poderá preencher total ou parcialmente alguns vazios entre as partículas de minério de ferro. O principal efeito do aumento da porosidade e da diminuição da quantidade de escória é a perda da resistência mecânica das pelotas, além do aumento da sua redutibilidade durante o processo de redução [3]. 
No caso de pelotas produzidas no Brasil, a importância relativa dessas várias características na resistência à degradação de pelotas não é, entretanto, ainda entendida com clareza. O presente trabalho visa analisar relações entre características mineralógicas e estruturais de pelotas selecionadas com a sua suscetibilidade à degradação física. Serão avaliados e relacionados aspectos da mineralogia, acabamento superficial, dureza da superfície, quantidade de poros, distribuição de tamanho de poros com medidas de resistência à compressão e abrasão, essa última obtida por meio de um ensaio modificado.

\section{MATERIAIS E MÉTODOS}

Amostras de quatro diferentes tipos de pelotas queimadas, comerciais, de minério de ferro produzidas em usinas brasileiras foram coletadas, tendo sido identificadas como A, B, C e D.

Foram realizadas análises químicas, através da técnica de fluorescência de raios $X$ (Espectrômetro de fluorescência de raios $X$ por energia dispersiva da marca Shimadzu Co), análises mineralógicas e medidas de porosidade, por meio da contagem de grãos em microscópio ótico (Microscópio Óptico Zeiss modelo Imager M2m, com auxílio do software de análise de imagens AxioVision 4.8) e microdureza na escala de Dureza Vickers (HV), por meio de microdurômetro Shimadzu DUH-211 Dynamic Ultra Micro Hardness Tester.

Também foram realizadas medidas de massa específica com picnômetro a Hélio e medida de porosidade no sistema microtomográfico de alta energia Skyscan 1173 (Skyscan/Bruker), no qual as projeções foram reconstruídas utilizando os programas Nrecon ${ }^{\circledR}$ SkyScan - versão 1.6.4.1 e InstaRecon - versão 1.3.5.0, para a análise de imagens.

Foram realizados ensaios de medida de resistência à compressão (ISO 4700) e tamboramento e abrasão (ISO 3271) seguindo os padrões adotados pela ISO.

De forma complementar, foram ainda realizados ensaios fragmentação superficial no tambor de abrasão do LTM, que é uma modificação do ensaio de tamboramento da ISO. O tambor utilizado neste ensaio possui o mesmo diâmetro (1 metro), porém com comprimento diferente ( 0,3 metros ao invés de 1 metro). Para a realização deste ensaio são utilizados dois lotes contendo 80 pelotas cada, escolhidas aleatoriamente dentro na faixa de $12,5 \times 9,0 \mathrm{~mm}$, e sendo suas massas balanceadas. Essa quantidade permite minimizar a interação entre as partículas, permitindo que o impacto ocorra majoritariamente contra a superfície interna do equipamento e não entre as pelotas. Dessa forma, o ensaio proposto [4] tem como objetivo analisar a fragmentação superficial de pelotas como resultado de impactos de baixa energia, medindo a abrasão.

\section{RESULTADOS E DISCUSSÃO}

\subsection{COMPOSIÇÃO QUÍMICA E ANÁLISE MINERALÓGICA}

A Tabela 1 apresenta os resultados das análises mineralógicas das 4 pelotas selecionadas. Como todas as pelotas analisadas são destinadas a fornos de redução direta e provenientes de minérios hematíticos, não há diferenças significativas nas composições químicas, com teores de ferro que variaram de 67,0 a 67,6\% e de sílica de 1,4 a $1,8 \%$. Os resultados médios das análises mineralógicas das amostras indicam que a pelota $D$ apresenta elevado teor de magnetita, que pode ter sido 
decorrente do excesso de queima, e pode acarretar em baixa resistência à compressão e baixo tamboramento das pelotas, enquanto as pelotas $A, B$ e $C$ apresentam indícios que foram queimadas sob boas condições.

Tabela 1 - Análise mineralógica média das amostras

\begin{tabular}{ccccc}
\hline \multirow{2}{*}{ Componente (\%) } & \multicolumn{5}{c}{ Pelota } \\
\cline { 2 - 5 } & A & B & C & D \\
\hline Hematita & 94,58 & 95,20 & 95,41 & 63,41 \\
Magnetita & 0,53 & 1,03 & 0,57 & 29,88 \\
Silicatos & 4,77 & 3,65 & 3,93 & 6,16 \\
Ferritos & 0,12 & 0,12 & 0,09 & 0,56 \\
\hline
\end{tabular}

As Figuras 1 a 4 apresentam um mosaico de imagens das microestruturas das pelotas analisadas. A pelota A apresenta pouca concentração de poros na região central e ausência de magnetita, enquanto que na região intermediária houve reoxidação da magnetita. A microestrutura da pelota B revela boas reações de sinterização, satisfatório crescimento de grão, pouca ocorrência de magnetita e muitos silicatos, sugerindo boa resistência física, devido a excelentes condições de queima. Outra evidência das excelentes condições de queima é que todas as fases microestruturais aparecem bem distribuídas em todas as regiões da pelota B. A pelota $\mathrm{C}$ apresenta microestrutura com elevada microporosidade e com macroporos detectados na região de extremidade, provenientes de insumos identificados nesta região. Já a microestrutura da pelota $\mathrm{D}$ apresenta porosidade concentrada na região central, onde também é identificada magnetita, enquanto que na região intermediária existe reoxidação da magnetita. Devido aos elevados tamanhos de grãos, ocorrência de grandes quantidades de magnetita e elevada porosidade na região central, pode-se afirmar que houve excesso de queima da pelota $D$, sugerindo baixa resistência mecânica para essa pelota.

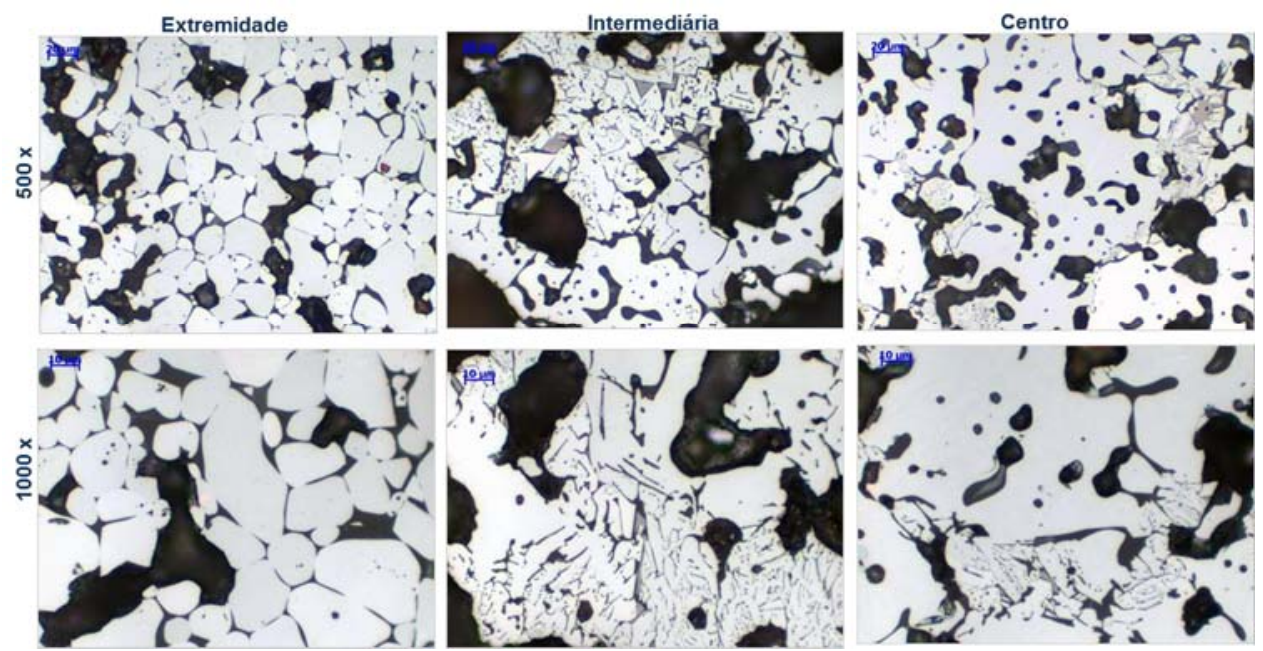

Figura 1 - Imagens da microestrutura da pelota A nas magnificações de 500x e 1000x, nas regiões de extremidade, intermediária e centro da pelota 


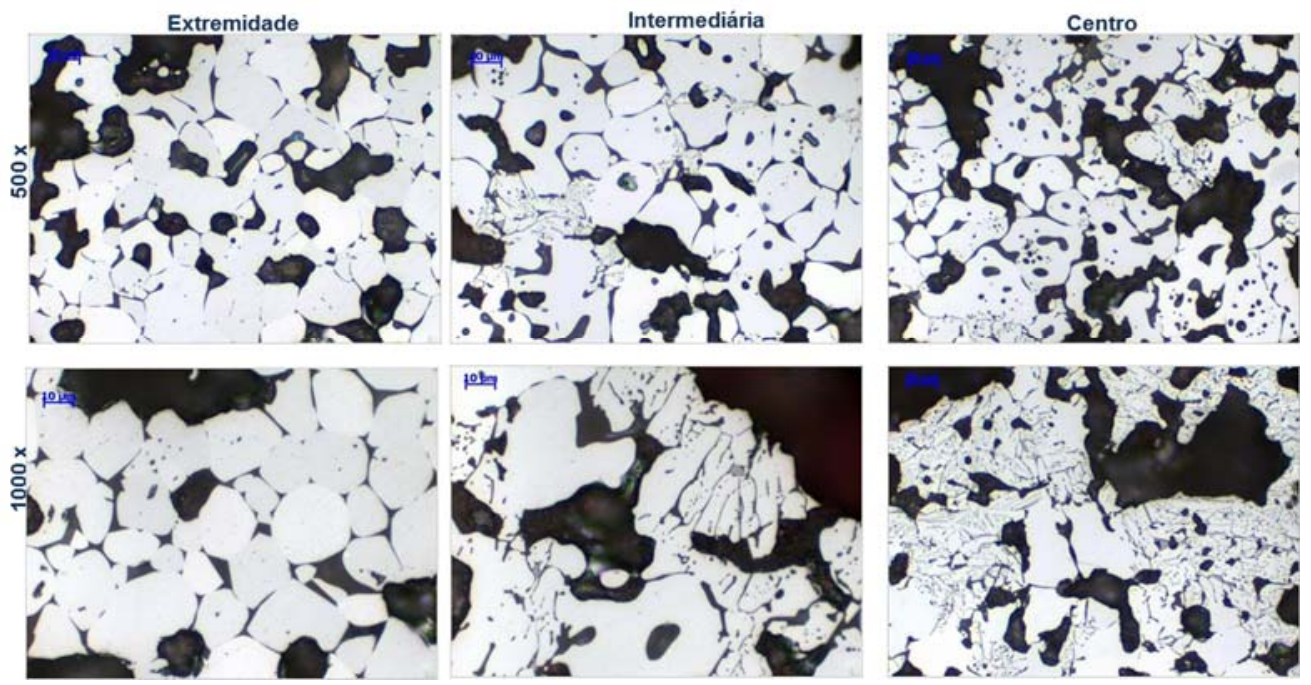

Figura 2 - Imagens da microestrutura da pelota B nas magnificações de 500x e 1000x, nas regiões de extremidade, intermediária e centro da pelota

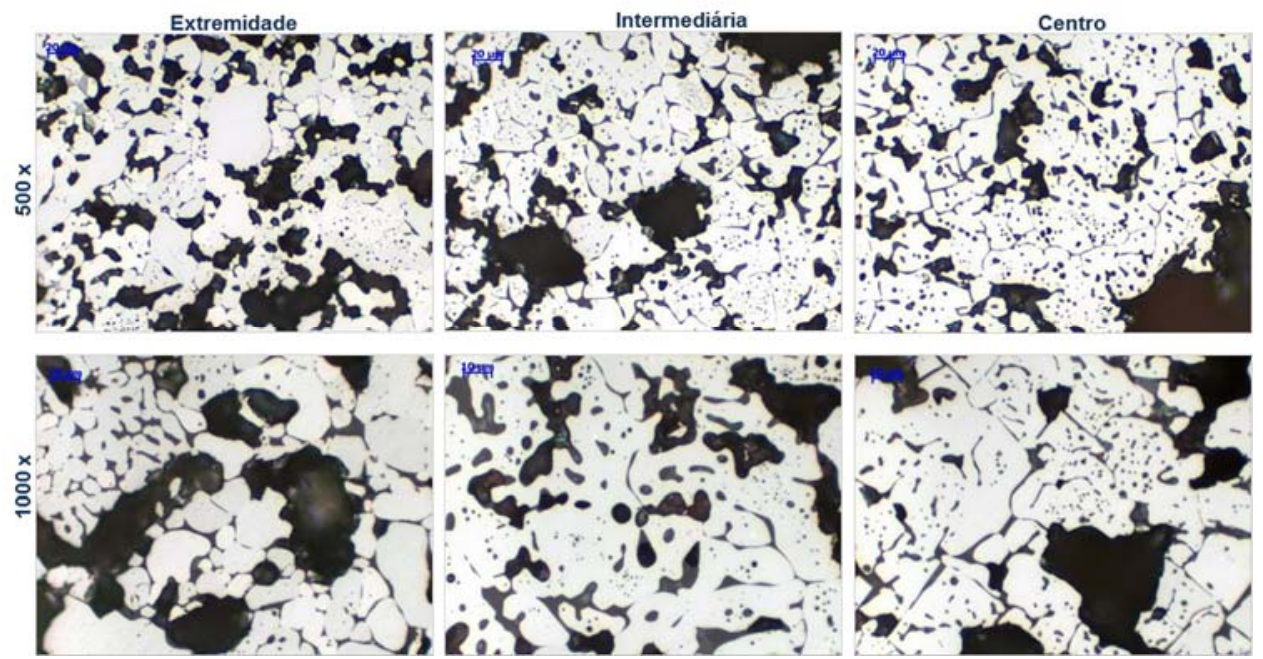

Figura 3 - Imagens da microestrutura da pelota C nas magnificações de 500x e 1000x, nas regiões de extremidade, intermediária e centro da pelota

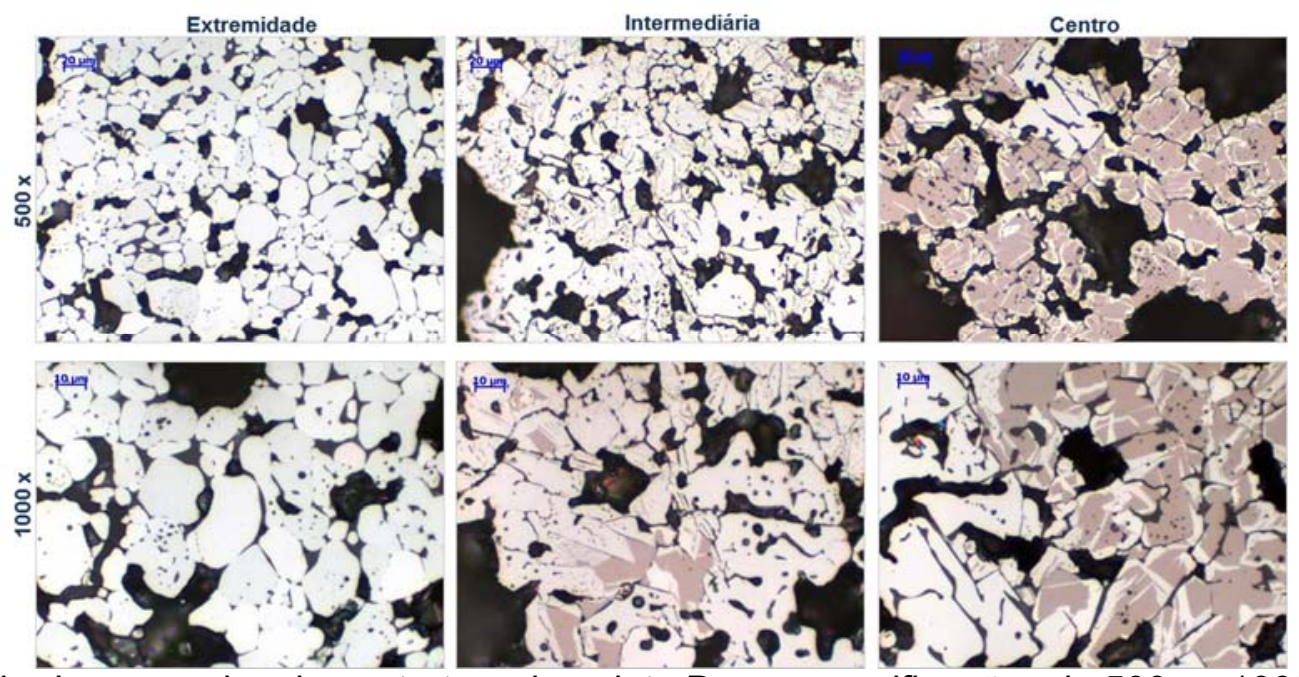

Figura 4 - Imagens da microestrutura da pelota D nas magnificações de 500x e 1000x, nas regiões de extremidade, intermediária e centro da pelota 


\subsection{POROSIDADE}

Os percentuais de porosidade foram medidos por diferentes técnicas, para cada pelota, sendo apresentados de forma sintética na Tabela 2. Na Tabela 2 são evidentes as grandes diferenças nos valores obtidos pelas diferentes técnicas. Apesar dessas diferenças, em todos os ensaios, a ordem da quantidade de poros foi crescente para as pelotas de A a D. No entanto, em alguns casos, essa diferença de porosidade é tão pequena que pode ser confundida com o erro experimental. $O$ ensaio considerado mais representativo foi o de medição de porosidade por meio da combinação das técnicas de Microtomografia Computadorizada de Raios- $X$ e Picnometria a Hélio. Apesar do ensaio de Microtomografia não possuir resolução alta o suficiente para, adequadamente, identificar os menores poros, por limitações do equipamento utilizado, esta técnica tornou possível estimar, de forma muito precisa, o volume das pelotas, identificando suas entrâncias e cavidades. Já a Picnometria a Hélio do material pulverizado forneceu valores precisos da massa específica da matriz da pelota, independente dos poros. Sendo assim, tem-se os valores precisos da densidade da matriz das pelotas, bem como o seu volume e massa aparentes. Dessa forma, acredita-se que os resultados deste ensaio sejam os mais próximos da realidade, tendo sido considerado o valor de referência quando comparado àqueles de outros ensaios realizados neste trabalho.

Tabela 2 - Resultados de medidas de porosidade para as amostras

\begin{tabular}{lcccc}
\hline \multirow{2}{*}{ Método } & \multicolumn{4}{c}{ Pelota } \\
\cline { 2 - 5 } & A & B & C & D \\
\hline Análise de imagens de microscopia (\%) & 40,0 & 42,6 & 43,5 & 49,6 \\
Microtomografia (\%) & 4,18 & 4,24 & 5,80 & 6,30 \\
Microtomografia e picnometria (\%) & 31,3 & 34,5 & 34,9 & 35,2 \\
\hline
\end{tabular}

Apesar de muito usado pelas usinas pelotizadoras, o cálculo da porosidade por meio da Análise de Imagens de microscopia de pelota não foi considerado muito representativo. Mesmo a análise sendo feita na pelota com resistência igual a média de resistência à compressão de cada amostra, a medição da porosidade é feita em uma única pelota, de maneira que não se avalia a variabilidade da amostra. Além disso, é realizada a análise apenas no corte transversal da pelota, onde estima-se a mesma porcentagem média de poros para o resto do volume, o que nem sempre é válido.

A Figura 5 ilustra um mosaico com as macroestruturas das amostras. A Pelota A apresenta excelente distribuição da porosidade, enquanto a Pelota B é pouco porosa com alguns macroporos, provavelmente, provenientes de insumos com granulometria grosseira na sua preparação. Já a Pelota $C$ apresenta algumas trincas finas, enquanto a Pelota $\mathrm{D}$ apresenta porosidade concentrada na região central. 


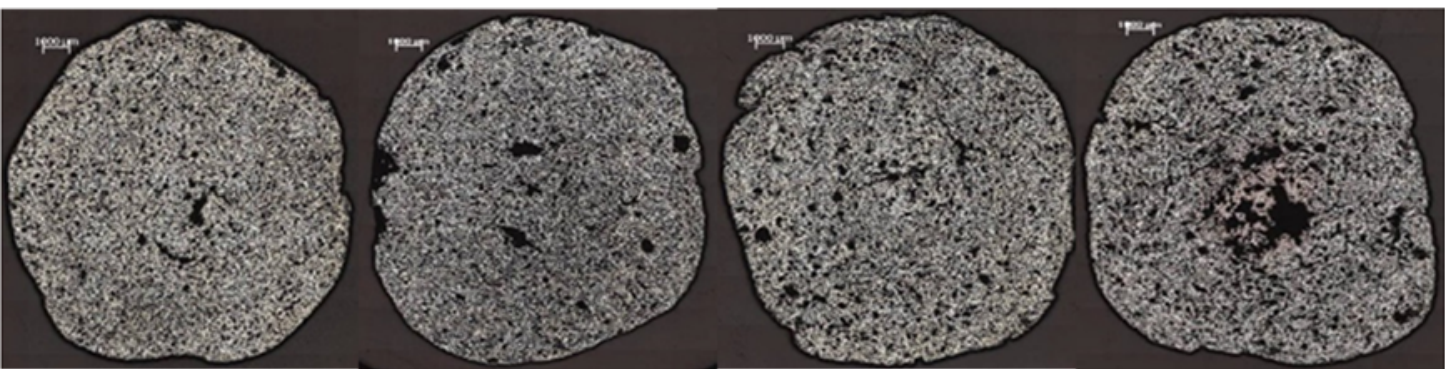

Figura 5 - Imagem da macroestrutura de pelotas selecionadas A, B, C e D, respectivamente, da esquerda para a direita.

Apesar de suas limitações em quantificar a porosidade total de pelotas, a Análise de Imagens de microscopia é uma ótima técnica para a análise de porosidade por região da pelota. A Tabela 3 apresenta esses resultados para as amostras analisadas. As pelotas $\mathrm{A}, \mathrm{B}$ e $\mathrm{C}$ apresentam uniformidade na porosidade das regiões analisadas, enquanto que a pelota $D$ mostra variação nos valores entre regiões, aumentando substancialmente a porosidade conforme se aproxima do centro. Maior porosidade na região central sugere menor resistência à fratura do material, o que faz com que a amostra $\mathrm{D}$ possua tendência a se comportar de maneira mais frágil.

Tabela 3 - Percentual de porosidade classificados por região das pelotas A, B, C e D

\begin{tabular}{ccccc}
\hline \multirow{2}{*}{ Região } & \multicolumn{5}{c}{ Pelota } \\
\cline { 2 - 5 } & $\mathrm{A}$ & $\mathrm{B}$ & $\mathrm{C}$ & $\mathrm{D}$ \\
\hline Extremidade & 40,7 & 42,9 & 44,7 & 45,1 \\
Intermediária & 38,1 & 41,2 & 42,7 & 49,9 \\
Centro & 41,0 & 43,7 & 43,1 & 53,9 \\
\hline Média & $\mathbf{4 0 , 0}$ & $\mathbf{4 2 , 6}$ & $\mathbf{4 3 , 5}$ & $\mathbf{4 9 , 6}$ \\
\hline
\end{tabular}

Apesar da limitada resolução do equipamento, as análises da porosidade por Microtomografia Computadorizada de Raios-X foram importantes para determinar as características geométricas dos poros, como diâmetro, área superficial e volume. É importante ressaltar que, devido ao limite de resolução, a técnica não permitiu a análise de todos os poros da pelota, tendo em vista que poros com diâmetros menores que, aproximadamente, $75 \mu \mathrm{m}$ não puderam ser detectados. As informações a respeito do espaço poroso das pelotas foram obtidas por meio das imagens reconstituídas (Figura 6), tendo tornado possível identificar apenas duas fases: matriz (sólida) e vazios (poros e trincas). 

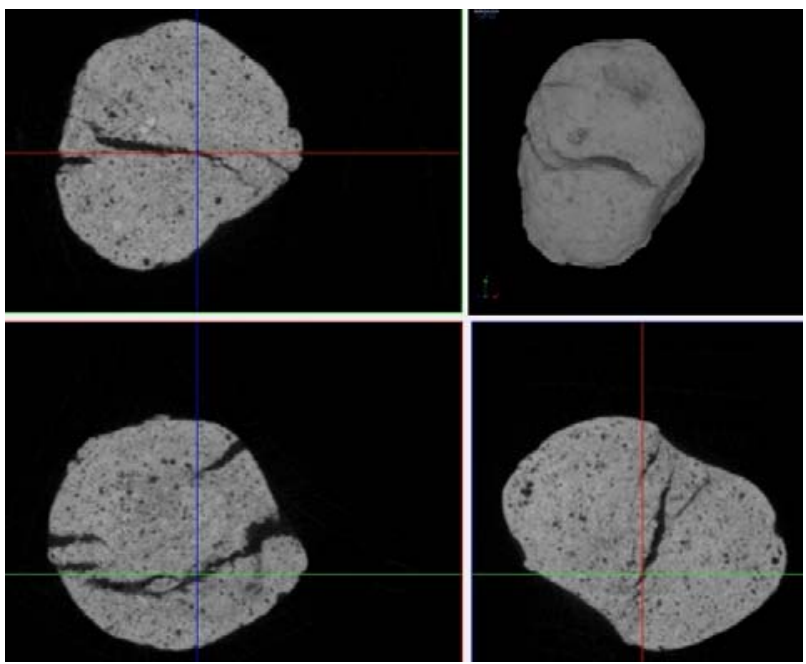

Figura 6 - Imagens reconstituídas da Pelota C em seções transversais e modelo 3D

A distribuição de tamanhos de poros foi determinada pela porcentagem do total de poros que a microtomografia foi capaz de analisar. Devido à baixa resolução do equipamento, a porosidade calculada pela microtomografia representa apenas uma parcela da porosidade total. Como mencionado, o valor considerado como referência, é o resultado da combinação das técnicas de Picnometria a Hélio e Microtomografia Computadorizada de Raios-X. Dessa forma, a porosidade calculada somente pela microtomografia subestimou a quantidade de poros das pelotas analisadas, indicando apenas os maiores poros e não a quantidade total destes. No entanto, o resultado deste ensaio indica o tamanho e volume dos poros, resultando em estimativas da distribuição volumétrica dos maiores poros das amostras das pelotas (aproximadamente dos 25\% maiores). Assim, a Figura 7 apresenta a distribuição volumétrica cumulativa de tamanho de poros, tendo em vista a porosidade total medida pelas técnicas de picnometria e microtomografia. Observa-se que as pelotas $C$ e $D$ possuem os poros com os maiores tamanhos, enquanto as pelotas $A$ e $B$ os menores. Isso sugeriria, a princípio, maiores resistências mecânicas das pelotas $A$ e $\mathrm{B}$ e valores mais baixos para as pelotas $\mathrm{C}$ e $\mathrm{D}$.

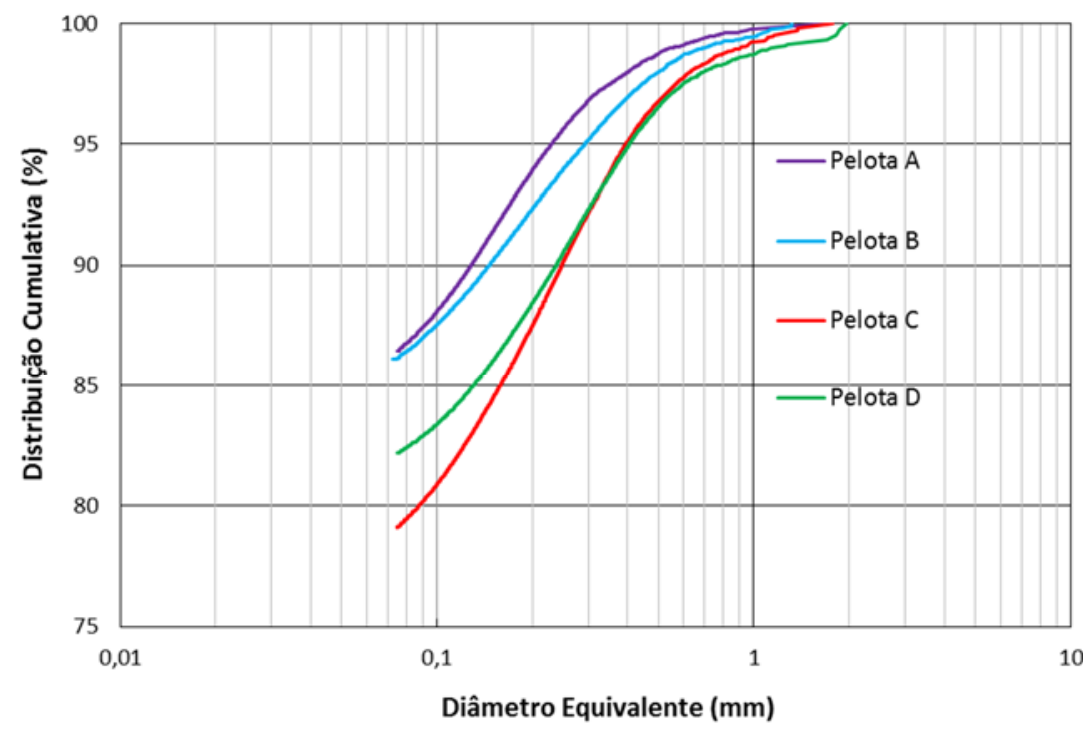

Figura 7 - Distribuição do tamanho de poros pelo volume cumulativo das amostras 
Assim, pode-se observar que, nos resultados dos ensaios de medição da porosidade, as pelotas $A$ e $B$ apresentaram quantidade e tamanho de poros menores que as pelotas $\mathrm{C}$ e $\mathrm{D}$. Além disso, as pelotas A e B apresentam maior homogeneidade na distribuição dos poros ao longo da amostra.

\subsection{ACABAMENTO SUPERFICIAL}

A Figura 5 ilustra um mosaico com as macroestruturas das pelotas $A, B, C$ e D, onde é possível analisar as bordas destas após o embutimento. Observa-se pequenas irregularidades nas bordas das pelotas $A$ e $B$, enquanto na pelota $C$ é observado alguns macroporos na região de extremidade e maiores irregularidades. Já na pelota D observa-se certa deformação.

Ao manusear as pelotas percebeu-se, por inspeção visual, que as pelotas da amostra A apresentam, em geral, bom acabamento superficial e arredondamento, com poucas trincas aparentes, enquanto que a amostra B apresentou certas irregularidades e algumas trincas de resiliência. O lote da amostra $C$ apresentou bom acabamento superficial e poucas trincas. Já na pelota $D$ foi possível observar certa porosidade superficial, assim como maior quantidade de trincas, além de pequenas deformações.

\subsection{ENSAIO DE MICRODUREZA}

A Tabela 4 apresenta os valores de microdureza das bordas (cavidades externas) e centros (cavidades internas) das amostras de pelotas, medidos por meio do ensaio de Microdureza Vickers. É evidente que as pelotas C e D obtiveram maiores valores de dureza nas bordas e os menores valores no centro, enquanto que a pelota $B$ apresentou baixos valores de dureza nas bordas. Observa-se que os valores do ensaio de microdureza nas pelotas apresenta coerência com os ensaios realizados por Huang [5], onde a microdureza das pelotas variou de 200 a $800 \mathrm{HV}$, aproximadamente.

Tabela 4 - Resultados médios de medidas de Microdureza do centro e das bordas das amostras

\begin{tabular}{ccc}
\hline Pelota & \multicolumn{2}{c}{ Dureza $(\mathrm{HV})$} \\
\cline { 2 - 3 } & Centro & Borda \\
\hline A & 880 & 727 \\
B & 545 & 335 \\
C & 288 & 883 \\
D & 533 & 810 \\
\hline
\end{tabular}

\subsection{QUALIDADE FÍSICA}

\subsubsection{Ensaios Padronizados (ISO)}

A Tabela 5 resume os resultados obtidos para as amostras que são objetivo do presente estudo, identificando-se diferenças consideráveis.

Os valores dos ensaios de Compressão Maior (pelotas com faixas de 16 x 12,5 mm), Compressão Menor (pelotas com faixas de 12,5 x $9 \mathrm{~mm}$ ), Tamboramento e Abrasão indicam essas diferenças na caracterização física, onde a pelota com maior resistência à compressão, junto com o maior índice de tamboramento, teoricamente, se degrada menos e a pelota com menores índices se degrada mais. Além disso, é observada uma relação diretamente proporcional entre a compressão e o 
tamboramento, sugerindo que o mesmo tipo de pelota que apresenta alta resistência à abrasão também apresenta alta resistência à fratura.

Tabela 5 - Resumo dos resultados dos ensaios de caracterização mecânica (Compressão,

Tamboramento e Abrasão) da ISO das pelotas A, B, C e D (Dados entre parênteses representam o Desvio Padrão dos resultados)

\begin{tabular}{ccccc}
\hline & A & B & C & D \\
\hline Compressão $16 \times 12,5 \mathrm{~mm}(\mathrm{kgf})$ & 357 & 322 & 300 & 260 \\
$<200 \mathrm{kgf}(\%)$ & $(107)$ & $(118)$ & $(124)$ & $(85)$ \\
$<150 \mathrm{kgf}(\%)$ & 17 & 16 & 21 & 45 \\
& 10 & 6 & 13 & 21 \\
\hline Compressão $12,5 \times 9 \mathrm{~mm}(\mathrm{kgf})$ & 300 & 299 & 288 & 239 \\
& $(154)$ & $(130)$ & $(125)$ & $(118)$ \\
$<200 \mathrm{kgf}(\%)$ & 34 & 36 & 39 & 62 \\
\hline Tamboramento & 18 & 18 & 25 & 44 \\
\hline Abrasão & 93,8 & 93,6 & 93,2 & 92,2 \\
\hline Abrasão padrão LTM (\%) & 5,5 & 5,7 & 5,9 & 6,6 \\
\hline
\end{tabular}

Observa-se que os resultados dos ensaios de resistência à compressão se mostraram coerentes com os resultados das análises mineralógicas. As pelotas $A$ e $B$ apresentaram melhores condições de queima, melhores distribuições das fases microestruturais em todas as regiões, menores quantidades de magnetita e, consequentemente, também apresentaram maiores valores de resistência à compressão, enquanto que a pelota $\mathrm{C}$ apresentou altas concentrações de silicatos na extremidade e também teor mais elevado de magnetita na extremidade. Na pelota $D$, observou-se uma concentração elevada de magnetita e tamanhos grandes dos grãos na região central, provavelmente, devido ao excesso de queima. Isso explica a baixa resistência à compressão dessa pelota em comparação às demais.

Também é possível constatar que a compressão das pelotas obedece a mesma tendência observada por Meyer [4], onde as pelotas com menor quantidade e tamanho de poros (Tabela 3 e Figura 5) apresentam maiores resistências à compressão (Tabela 5).

Verifica-se que, apesar da avaliação com caráter subjetivo, o acabamento superficial aponta para a mesma tendência dos resultados das análises mineralógicas e dos ensaios de compressão e tamboramento propostos pela ISO.

No entanto, os resultados da microdureza indicam maior dureza da borda nas pelotas $\mathrm{C}$ e $\mathrm{D}$, enquanto a pelota $\mathrm{B}$ apresenta valores consideravelmente mais baixos, o que se diferencia da compressão. Acredita-se que essa dureza tenha relação com o percentual de silicatos das extremidades das pelotas. Já os resultados para os testes de microdureza no centro das pelotas, também não se observa relação com os resultados da resistência à compressão. Também se observa certa relação com o percentual de silicatos no centro das pelotas, porém com o possível efeito sendo compensado pela grande quantidade de magnetita na região central da pelota $\mathrm{D}$.

\subsubsection{Fragmentação Superficial (Tambor de Abrasão LTM)}

O ensaio de fragmentação superficial, realizado no tambor de abrasão do LTM, indica o quanto cada pelota perde de massa a cada impacto de baixa magnitude. A Figura 8 apresenta os dados da relação de perda mássica por impacto com a dureza da borda das diferentes pelotas. Observa-se que há excelente correlação da fragmentação superficial (perda mássica por impacto de baixa magnitude) com a dureza na borda 
das pelotas. Não houve correlação entre a perda mássica por abrasão e os ensaios de tamboramento e compressão propostos pela ISO.

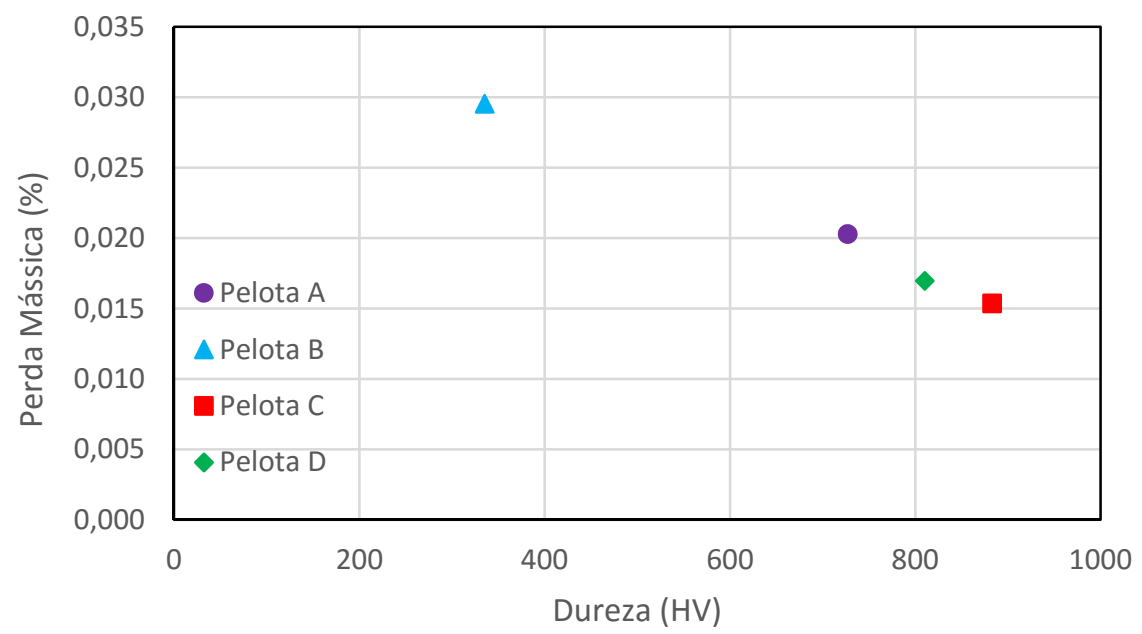

Figura 3.4 - Relação entre a Dureza da borda das pelotas A, B, C e D com sua fragmentação superficial

\section{CONCLUSÕES}

Os resultados deste estudo concluem que há excelente correlação das características mineralógicas e estruturais das pelotas com sua suscetibilidade à degradação mecânica.

As análises mineralógicas foram capazes de sugerir sobre as condições de queima e, consequentemente, sobre a resistência à compressão das pelotas. A análise do acabamento superficial mostrou-se coerente com os resultados de resistência à compressão. Apesar da sua subjetividade, ela se apresenta como um método de avaliação das pelotas com boa relação custo benefício, sendo uma excelente ferramenta de avaliação preliminar.

A quantidade e o tamanho máximo dos poros presentes nas pelotas também apresentou forte correlação com a resistência à compressão. Como observado na literatura [3], quanto maior a quantidade e o tamanho dos vazios (poros), menor a resistência mecânica das pelotas. No entanto, essa relação não foi mantida em relação à porosidade da extremidade com a suscetibilidade à abrasão. Supostamente a pelota com maior porosidade na extremidade deveria ser a que mais perderia massa por abrasão. Porém, o observado foi exatamente o contrário. As pelotas C e D apresentaram maiores valores de porosidade na superfície e também os menores valores de perda mássica por impactos de baixa magnitude. Desta forma, a porosidade não apresenta a mesma relação com a resistência à compressão e com a suscetibilidade à abrasão.

Pôde-se concluir também que, como mencionado anteriormente, a perda mássica por abrasão não tem relação com os ensaios de tamboramento e abrasão propostos pela ISO. Entretanto, os ensaios de fragmentação superficial propostos apresentaram excelente correlação com a dureza superficial. Acredita-se que a dureza medida na extremidade das pelotas tenha relação direta com o percentual de silicatos na região. Sabe-se que ainda são necessários mais testes para comprovar se a medição da dureza superficial pode ser usada como índice de suscetibilidade à abrasão, mas já se tem um bom indicativo. 


\section{Agradecimentos}

Os autores agradecem ao Laboratório de Instrumentação Nuclear da Universidade Federal do Rio de Janeiro pelos ensaios de Microtomografia Computadorizada de Raios-X.

\section{REFERÊNCIAS}

1 FONSECA, M. C., 2004, Influência da distribuição granulométrica do pellet feed no processo de aglomeração e na qualidade da pelota de minério de ferro para redução direta. Dissertação de Mestrado. REDEMAT. Universidade Federal de Ouro Preto, Ouro Preto.

2 BAILON, A. M. G., SIMÕES, H.O., ANACLETO, A.J., ALMEIDA, R.R., PEDRUZZI, A., FONSECA, M.C., PASSIGATI, V.P., Avaliação de fases microestruturais e da resistência a compressão de pelotas de minério de ferro producidas em diferentes temperaturas de queima, $2015,70^{\circ}$ Congresso Anual ABM, Rio de Janeiro.

3 MEYER, K., 1980, Pelletizing of Iron Ores, Springer-Verlag Berlin, Heidelberg, Germany.

4 SILVEIRA, M.A.C.W., 2012, Modelagem da degradação de pelotas de minério de ferro durante o manuseio e transporte, Dissertação de Mestrado. Curso de Pós-Graduação em Engenharia Metalúrgica e de Materiais, COPPE/UFRJ, Rio de Janeiro.

5 HUANG, Z.; YI, L.; JIANG, T., 2012, Mechanisms of strength decrease in the initial reduction of iron ore oxide pellets. Powder Technology, v. 221, p. 284-291. 